\title{
La enseñanza en la educación secundaria
}





\title{
La regulación metacognitiva sobre los modos de pensar en el aula de biología
}

( Gastón Mariano Pérez

Consejo Nacional de Ciencia y Tecnología / Universidad de Buenos Aires, Argentina gastonperezbio@gmail.com

\author{
Leonardo Martín González Galli \\ Consejo Nacional de Ciencia y Tecnología / Universidad de Buenos Aires, Argentina \\ leomgalli@gmail.com
}

\begin{abstract}
Resumen
La enseñanza y el aprendizaje de las ciencias suele ser problemático debido a múltiples factores. Uno de estos problemas corresponde a los modos de razonar u obstáculos epistemológicos de los sujetos como pueden ser, entre otros, el esencialismo o el determinismo. En este trabajo nos propusimos caracterizar los procesos de regulación metacognitiva que emergieron durante una propuesta didáctica basada en la modelización y la metacognición sobre los obstáculos epistemológicos. Esta caracterización se basó en los datos colectados de una secuencia didáctica sobre dos modelos de la biología evolutiva. Desde una perspectiva cualitativa-interpretativa se construyeron tres categorías para cumplimentar con el objetivo. Una de ellas corresponde a un tipo de regulación simple en la que solo se señala el obstáculo epistemológico bajo ciertas explicaciones de los estudiantes. Las otras dos categorías corresponden a una regulación de tipo compleja en la que además de señalar se construye una explicación alternativa con elementos construidos durante la secuencia didáctica. En esta última distinguimos regulaciones de tipo individual y de tipo social. Estas categorías permiten pensar en posibles intervenciones con el objetivo de desarrollar habilidades metacognitivas necesarias para construir un pensamiento crítico en los estudiantes.
\end{abstract}

Palabras clave: Regulación metacognitiva, obstáculos epistemológicos, biología evolutiva, determinismo, esencialismo 


\title{
The metacognitive regulation on ways of thinking in the biology classroom
}

\begin{abstract}
The teaching and learning of science is often problematic due to multiple factors. One of these problems corresponds to the ways of thinking or epistemological obstacles such as, among others, essentialism or determinism. In this paper, we set out to characterize the processes of metacognitive regulation that emerged during a teaching proposal based on modeling and metacognition on epistemological obstacles. This characterization was based on the data collected from the sequence for teaching two models of evolutionary biology. From a qualitative-interpretative perspective, three categories were constructed to fulfill the objective. One of them corresponds to a simple type of regulation in which only the epistemological obstacle is pointed out under certain explanations of the students. The other two categories correspond to a complex type of regulation in which, in addition to pointing out, an alternative explanation is constructed with elements constructed during the didactic sequence. In the latter, we distinguish individual and social type regulations. These categories allow us to think about possible interventions to develop the metacognitive skills necessary to build critical thinking in students.
\end{abstract}

Keywords: Metacognitive regulation, epistemological obstacles, evolutionary biology, determinism, essentialism

\section{Introducción}

En la actualidad diversas investigaciones manifiestan que $\operatorname{los}^{1}$ estudiantes de nivel medio terminan sus estudios obligatorios con una baja comprensión de los contenidos científicos que se les enseñan. En este sentido podemos citar los resultados del operativo nacional de evaluación ONE del año 2013 que propuso que solo 13 de cada 100 estudiantes del último año de la secundaria lograban resolver problemas y realizar predicciones, transfiriendo estrategias y saberes desde otros contextos.

Resultados de este estilo pueden ser explicados por múltiples factores, uno de los cuales puede ser la persistencia de las concepciones, alternativas a los modelos científicos, que sostienen los estudiantes a pesar de la instrucción (Astolfi, 2003; Carretero, 2009; Johsua y Dupin, 2005; entre otros). Esto implica que ante la resolución de problemas o la toma de decisiones sobre cuestiones de la vida cotidiana, los sujetos optan por utilizar sus concepciones del sentido común en desmedro de los modelos científicos. El caso más visible por el que transitamos hoy en día son los engaños de las llamadas "pseudo-ciencias" así como las diferentes propuestas de los medios de comunicación (publicidades, propagandas, noticias) controlados por los grupos que detentan el poder económico y político.

Es importante destacar que el conocimiento cotidiano relativo a cuestiones de salud, ambiente, consumo, etc., puede ser útil para algunas situaciones pero en general supone una mirada simplista de la realidad. Esto es problemático dado que lleva a tomar decisiones con consecuencias nefastas, no solo en el plano individual del sujeto que decide sino

1 Optamos por esta forma de escritura, para una fácil lectura, aunque somos conscientes de que dejan por fuera muchas expresiones de género conocidas o por conocer. 
en el plano social. Tomemos como ejemplo el actual auge de los grupos anti-vacunas y el aumento de casos de Sarampión con complicaciones graves en distintas partes del mundo; o el caso del uso de agrotóxicos y el envenenamiento de las poblaciones. La utilización de los conocimientos científicos para tomar decisiones sobre estos y otros temas tendría claras consecuencias diferentes (García, 1998; Pozo, 2016; Solbes, 2013).

Frente a esta situación es clave el desarrollo del pensamiento crítico que incluya, en la enseñanza de las ciencias, la construcción de modelos científicos útiles para los estudiantes. No obstante, fomentar el pensamiento crítico en el aula no sería una tarea fácil, si entendemos que es una habilidad de pensamiento compleja que involucra en sí otras habilidades. Una de ellas es la metacognición, que implica una conciencia y una regulación sobre los modos de pensar de los sujetos. Fomentar la metacognición en el aula involucraría, entre otras cosas, desarrollar juicios reflexivos, la habilidad de auto-corrección, la reflexión sobre el conocimiento que está implícito en la acción, el reconocimiento de las propias capacidades y limitaciones, así como la explicitación y discusión de los sesgos cognitivos (López Aymes, 2012; Norabuena et al., 2017). De esta manera, fomentar procesos metacognitivos en el aula aportaría al desarrollo de un pensamiento crítico (Ford y Yore, 2012; Hogan et al., 2015; Tamayo Alzate, 2014; Torres y Solbes, 2018).

En función de lo expuesto hasta el momento, cabría preguntarse de qué manera fomentar en el aula procesos metacognitivos y cuál sería su alcance. Para comenzar a delinear una posible respuesta, presentaremos en este artículo algunos resultados del trabajo doctoral del primero de los autores que versó sobre la enseñanza y el aprendizaje de los modelos evolutivos en el marco de una secuencia didáctica basada en la modelización y en la metacognición sobre los obstáculos epistemológicos. Más particularmente, nos centraremos en el análisis de los procesos de regulación metacognitiva que emergieron durante dicha propuesta.

En una primera instancia expondremos algunos aspectos del marco teórico que permitirán comprender el análisis de los datos. Luego, de manera breve, comentaremos las cuestiones metodológicas del trabajo para posteriormente presentar algunos resultados junto con su discusión. Concluiremos con algunas ideas sobre el desarrollo de la metacognición en las aulas de nivel medio.

\section{La regulación de los modos de pensar intuitivos}

Según diversos autores de la psicología cognitiva, los sujetos tomamos decisiones a partir de un sistema de dos vías: una ruta intuitiva y una razonada (Evans, 2018; Evans y Rosengren, 2018; Fletcher y Carruthers, 2012; Hogan et al., 2015). La ruta intuitiva implica un procesamiento de rápida respuesta que es inconsciente, automática y guiada por principios que son, en alguna medida, innatos. La ruta razonada corresponde al juicio reflexivo de carácter lento, consciente y asociado a altos niveles de control cognitivo. Posee principios que varían entre los individuos y las culturas. Ambos tipos de razonamiento trabajan en paralelo, y por lo tanto son capaces de llegar a decisiones diferentes. Teniendo en cuenta que el juicio intuitivo funciona automáticamente, los errores asociados y prejuicios pueden ser prevenidos a través de la regulación que permite el juicio reflexivo. Por lo tanto, un desafío clave en la enseñanza es el cultivo de habilidades de autorregulación que apoyen la conciencia metacognitiva y el control que a menudo son necesarias para el juicio reflexivo de alto nivel, así como el pensamiento crítico.

Para ciertos autores, de la misma manera que manejar un auto comienza siendo una actividad demandante para el sujeto y que tras mucha práctica es automatizada, lo mismo podría ocurrir con algunos procesos de la ruta razonada. La investigación actual 
muestra que el aprendizaje en cualquier dominio no es una progresión lineal desde el sistema intuitivo a un razonamiento científico, sino que existen múltiples caminos en los que ambos sistemas trabajan juntos (Evans, 2018; Evans y Rosengren, 2018; Halpern, 2013; Pozo, 2014, 2016).

Para Pozo (2016) la mayoría de las personas ignoramos que nuestra mente funciona así, por lo que no podemos controlar tanto como creemos nuestras ideas y acciones, pero quienes diseñan los mensajes publicitarios, quienes difunden ideología o consignas políticas sí lo saben. En este sentido, para el autor aprender ciencias no implicaría solamente proporcionar a los aprendices saberes establecidos, sino que es necesario en primer lugar que tomen conciencia de sus modos de razonar intuitivos y en segundo lugar que desarrollen la capacidad de controlarlos.

Esta propuesta se asocia al desarrollo del pensamiento crítico, en tanto que el mismo involucraría procesos de auto-corrección sobre los modos de pensar (Ford y Yore, 2012; Hogan et al., 2015; Tamayo Alzate et al., 2017).

Para avanzar en la distinción entre la ruta intuitiva y la ruta razonada de toma de decisiones, propondremos a continuación dos ejemplos de modos de razonar intuitivos de los sujetos: el esencialismo y el determinismo.

\section{Dos ejemplos de modos de razonar intuitivos}

En las clases de biología podemos escuchar expresiones como: "Las personas y algunos otros animales como los perros sí son todos distintos, pero en el caso de las hormigas o las bacterias no suele haber grandes diferencias" o "De un pez no puede haber evolucionado un lobo". Estas ideas pueden entenderse como expresiones de un tipo de razonamiento denominado esencialista.

Una de las definiciones más descriptivas (e interesantes para la enseñanza) sobre el esencialismo es la propuesta por Susan Gelman y Marjorie Rhodes (2012). Estas investigadoras proponen que el razonamiento esencialista está basado en cinco supuestos:

(1) Supuesto de estabilidad e inmutabilidad de la esencia. Implica pensar que las categorías que construimos son estables e inmutables. Aunque los miembros de cada categoría cambien exteriormente, esta variación es superficial con la esencia subyacente no modificada. En otras palabras, los miembros no pueden cambiar de categoría.

(2) Supuesto de límites intensificados. Asume que los límites entre las categorías son estrictos e impermeables.

(3) Supuesto de variabilidad como ruido. Las categorías se representan de manera homogénea en términos de una estructura prototípica. En este sentido, aquellas variaciones entre los miembros de la categoría se tratan como meramente superficiales.

(4) Supuesto de causas inherentes a los individuos. Involucra pensar que existe algo interno (esencia) en cada individuo que causa que tenga las características y comportamientos que tiene. Es esta esencia la que todos los miembros de la categoría comparten y funciona como una fuerza causal individual.

(5) Supuesto de la noción platónica de idea. Se asume que la esencia es un ideal al que nunca puede accederse en el mundo real. Así, la esencia correspondería a lo ideal y nunca se puede acceder a ella directamente. 
El esencialismo, como forma de razonamiento posee muchas ventajas. Por ejemplo, podría ser la base de nuestra capacidad para clasificar y hacer inferencias a partir de esas categorías. De esta manera, cuando se nos habla sobre algo que podemos haber categorizado en nuestra cabeza (por ejemplo: categoría perro, categoría docente), esta habilidad de categorización reduce el número de información que tenemos que procesar para dar una respuesta. Si así no fuera, cada vez que nos encontráramos con un perro o un docente no podríamos anticipar o entender sus acciones, siendo algo nuevo para nosotros, tal como le ocurría a Ireneo Funes del cuento de Borges ("Funes, el memorioso").

Además, al concebir la variación como algo superficial y sin importancia, permite enfocarse en las propiedades generales, limitando la composición de nuestras representaciones mentales sobre la categoría y sus miembros (Atran et al., 2001; Coley y Muratore, 2012; Evans, 2008).

Por otro lado, el modo de razonar esencialista es la base de la construcción de categorías sociales, como clase social, razas o género. Los sujetos tienden a considerar la pertenencia a una categoría social como el reflejo de una identidad única y real. Para estos casos también rige la inalterabilidad de la categoría, así como la heredabilidad de la esencia. Claramente esto lleva aparejado en nuestra sociedad sesgos racistas, sexistas, de clase (Del Río y Strasser, 2007; Hirschfeld, 2002; Mayr, 2006; Sperber, 2002).

El otro modo de razonar sobre el que vamos a discutir es el determinista. Podemos decir que implica pensar que el estado actual de un sistema determina su estado futuro, sin una asignación de probabilidades a los posibles estados futuros alternativos. De esta manera, aquello que "es" no cabe la posibilidad de que sea de otra manera. Ejemplos de expresiones basadas en este razonamiento en la clase de biología pueden ser: "Solo los organismos más aptos son los que sobrevivirán a través del tiempo" o "La violencia la lleva en los genes".

Este modo de razonar es funcional para cualquier sujeto, dado que permite reducir o suprimir la incertidumbre de las cosas, así como tener la capacidad de pronosticar de manera certera (Tversky y Kahneman, 1974). Si en cambio se asignaran probabilidades a todos los posibles eventos, no se podría predecir un futuro. El sistema educativo tradicionalmente introduce desde los primeros años de escolarización una visión determinista del mundo (por ejemplo: la física newtoniana) en forma de leyes de "obligado cumplimiento", lo que también contribuye a la funcionalidad de esta forma de pensar en los contextos escolares.

Sin embargo, de la misma manera que el esencialismo es la base de importantes sesgos, el determinismo también lo es. Puede encontrarse en expresiones históricas de pensadores como Aristóteles o Platón, donde la alusión a un Dios conducía a concebir todos los procesos como previamente determinados, hasta en los discursos públicos relacionados con el determinismo biológico o con el determinismo cultural (Puig y Jiménez Aleixandre, 2015; Stamos, 2008).

En función de todo lo expuesto, particularmente de los sesgos que traen aparejados tanto el esencialismo como el determinismo, cabe insistir en la importancia de habilitar en el aula espacios para la reflexión sobre estos modos de pensar.

\section{La influencia de estos modos de razonar en el aprendizaje de la biología evolutiva}

En este apartado nos centraremos en la enseñanza de la biología evolutiva. Es importante destacar que comprender los modelos de la biología evolutiva permitiría tomar 
decisiones o comprender cuestiones como el control y tratamiento de infecciones, la resistencia bacteriana a los antibióticos, la distribución del VIH, entre otras. Pero además, la perspectiva evolutiva permite explicar cómo pensamos acerca de la normalidad y la anormalidad, o sobre las diferencias en las características humanas (Dennett, 1999; Dupré, 2006; Futuyma, 2009; Stamos, 2008).

Los modos de pensar que mencionábamos en el apartado anterior influyen fuertemente en los aprendizajes sobre los modelos evolutivos. En el caso del esencialismo, los supuestos allí expresados son incompatibles con distintos modelos de la biología evolutiva como el de selección natural o especiación alopátrica. Por ejemplo, el supuesto de estabilidad de una categoría es incongruente con la teoría evolutiva que se basa en que las especies cambian a través de las generaciones. Por su lado, el supuesto de variabilidad como ruido, que implica ignorar o desestimar la importancia de la variabilidad, es un problema en dos sentidos en la comprensión del modelo de selección natural: primero, porque la variabilidad es necesaria para que la selección natural actúe; y segundo, aunque uno asuma la variabilidad, una perspectiva esencialista tiende a considerarla superficial con la esencia subyacente intocable. Finalmente, el esencialismo lleva a realizar análisis de tipo individual y no a nivel poblacional, que es el nivel en el que se explican los cambios evolutivos. De esta manera, se piensa que el rasgo esencial o naturaleza (por ejemplo, la felinidad peculiar de los tigres) subyace a los rasgos perceptibles mejor conocidos (por ejemplo, ser grande y tener rayas); es decir, las características perceptivas de una especie se presume que son consecuencias naturales de la esencia de ese tipo, aunque la naturaleza esencial es en gran parte desconocida y quizás efectivamente incognoscible (Atran et al., 2001; Waxman, Medin, y Ross, 2007).

Por su parte, para el caso del determinismo, este modo de razonar provoca que se entiendan los modelos evolutivos como un "todo o nada" con todos los individuos "no adaptados" muriendo y los "adaptados" sobreviviendo. Para los estudiantes, la evolución es la antítesis de la probabilidad. Los hechos sucedidos se consideran más bien como inevitables y no como una de las posibles líneas de evolución. Otras expresiones del determinismo pueden interpretarse desde las ideas del sentido común de los sujetos sobre la evolución de los humanos, mientras que la gran mayoría conciben al humano como fin y coronamiento necesario de la evolución (Gregory, 2009; Mead y Scott, 2010). Así, el determinismo impide comprender que procesos evolutivos como la selección natural son consecuencia de diferencias probabilísticas en la supervivencia de individuos con distintas variantes de un rasgo, que se originaron de manera azarosa.

Lo que se espera es que, como contrapunto de estos modos de razonar, los estudiantes construyan los siguientes postulados: que se percibe una variación continua en la naturaleza; que se percibe una ubicua variación interindividual y esta es relevante para la evolución; que las nuevas especies se originan como lo que inicialmente eran variedades de otras especies; y que la selección natural es consecuencia de diferencias probabilísticas en la supervivencia de individuos con distintas variantes de un rasgo.

\section{La enseñanza de la biología evolutiva basada en la regulación de los modos de pensar}

Una posible propuesta didáctica, para abordar la reflexión sobre estos modos de razonar en el aula, es la que proponen didactas franceses como Astolfi y Peterfalvi (2001), Johsua y Dupin (2005) o Martinand (1986). Estos autores trabajan sobre el concepto de obstáculo epistemológico, originalmente propuesto por el filósofo Gastón Bachelard, para quien los obstáculos son entendidos de manera fuertemente negativa, como obstáculos 
a destruir. Sin embargo, para los didactas franceses, los obstáculos epistemológicos son marcos de pensamiento o modos de razonar que conceden a la mente una facilidad para pensar de manera sencilla (Astolfi, 2003).

Así, desde esta perspectiva (que coincide con los trabajos mencionados de la psicología cognitiva), un obstáculo epistemológico nunca se resuelve definitivamente; las posibilidades de eliminarlos o modificarlos radicalmente serán limitadas. Tampoco sería deseable esta eliminación si consideramos que estos modos de pensar constituyen rasgos funcionales y constitutivos de la cognición humana, como indicamos previamente en los ejemplos del esencialismo y el determinismo. Entonces se espera propiciar en los sujetos una reflexión metacognitiva sobre estos modos de pensar, que incluya una consciencia sobre ellos y también una regulación de los mismos cuando se está construyendo o utilizando un modelo científico (Peterfalvi, 2001; González Galli y Meinardi, 2015, 2016, 2017; González Galli et al., 2018).

Las actividades que se construyan para desarrollar estas habilidades en el aula pueden ser de índole diversa (para algunos ejemplos, ver Astolfi y Peterfalvi, 2001; González Galli, 2011; González Galli et al., 2018). Generalmente se espera que sean actividades donde se trabaje de manera explícita el modo de razonamiento, visibilizando en el aula cómo son, de qué manera circulan en la sociedad y cómo influyen en los aprendizajes. Este tipo de actividades cumplen un doble rol: por un lado, permiten razonar sobre la comprensión de los modelos científicos y, por otro lado, permiten transformar en objeto de discusión esos modos de pensar más allá de la disciplina científica que se está enseñando. Comprender que estos razonamientos son transversales a múltiples dominios del conocimiento es una tarea difícil, sin embargo, trabajarlos de manera local en una disciplina como puede ser la biología evolutiva es un aporte significativo que apunta en ese sentido.

\section{Aspectos metodológicos}

El presente trabajo se incluye en el proyecto de tesis doctoral del primero de los autores. La tesis se encuentra enmarcada dentro del paradigma cualitativo-interpretativo y posee algunos elementos del diseño de investigación correspondiente a la teoría fundamentada (Creswell, 2012; Flick, 2007). El objetivo general de la tesis es contribuir a la comprensión de los procesos de construcción de explicaciones basadas en los modelos de evolución por selección natural y especiación alopátrica. De este objetivo se desprende el objetivo específico propuesto para este trabajo: caracterizar los procesos de regulación metacognitiva que emergieron durante la propuesta didáctica.

Desde el enfoque de investigación adoptado se espera producir una explicación respecto de un fenómeno, la cual está basada en los datos recolectados en el campo. El marco teórico cumple el rol de orientador del trabajo en terreno, lo que admite la emergencia gradual de preguntas y conceptos desde categorías cercanas al material empírico, hasta llegar a un alto grado de abstracción. El pasaje desde aquellas categorías más cercanas a la empiria hasta aquellas más abstractas es posible cuando el investigador construye, en un movimiento en espiral -de la empiria a la teoría y de la teoría a la empiria-, sus categorías y proposiciones teóricas (Glaser y Strauss, 1967; Strauss y Corbin, 1990).

Los registros sobre los que se construyeron las categorías provienen de la implementación de una secuencia didáctica formada por 21 actividades, basada en la modelización y la metacognición sobre los obstáculos epistemológicos. La propuesta didáctica estuvo orientada a la construcción de dos modelos de la biología evolutiva: el modelo de evolución por selección natural y el modelo de especiación alopátrica. 
Con el objetivo de que los estudiantes aprendan a regular los obstáculos epistemológicos, durante la secuencia didáctica se realizaron diversas actividades. Las mismas involucraron la identificación, por parte de los estudiantes, de los obstáculos epistemológicos en producciones propias o ajenas. En algunos casos, luego de identificar estos modos de razonar, se consensuó con la clase una etiqueta lingüística que permitiera evocar los obstáculos siempre que fuera necesario. Se establecieron instancias donde los estudiantes debían reformular las explicaciones, elaboradas por ellos, en las que los obstáculos epistemológicos implicaban alguna contradicción con el modelo científico. También se propuso volver sobre las producciones anteriores, de manera de hacerse conscientes sobre los obstáculos epistemológicos presentes y poder regularlos utilizando los modelos científicos construidos como una referencia teórica para dicha regulación.

Todo este trabajo implicó un andamiaje por parte del docente. Inicialmente se ayudó a los estudiantes a identificar y regular los obstáculos epistemológicos a partir de ofrecer una primera definición y evidenciar su funcionalidad en la vida cotidiana, su transversalidad en diversos contextos y dominios de conocimiento, y su conflictividad con los modelos científicos a aprender. Este andamiaje, a medida que avanzó en la secuencia, fue dando lugar a otras modalidades de trabajo más autónomas, algunas de las cuales implicaban instancias de regulación entre los propios compañeros.

Un mayor detalle de la secuencia didáctica puede encontrarse en Pérez, Gómez Galindo y González Galli (2018).

El análisis de los datos se realizó a partir del método comparativo constante (Glaser y Strauss, 1967; Strauss y Corbin, 1990) con el objetivo de encontrar regularidades en los registros colectados, las que se nombran creando categorías teóricas que tienen sentido para responder a las preguntas de investigación. Posteriormente se continuó buscando más evidencia que sustentase estas categorías, lo que devino en una comparación constante entre categorías y nuevos datos de la empiria.

De esta investigación participaron 75 estudiantes provenientes de una escuela de educación media de gestión pública de la Ciudad Autónoma de Buenos Aires (CABA). Dicha institución recibe estudiantes de clase media provenientes tanto de la CABA como de la provincia de Buenos Aires. Los grupos-clase que participaron de la investigación estaban compuestos por entre 23 y 29 estudiantes pertenecientes al segundo año de la escuela media ( 14-15 años). La secuencia didáctica implementada para la toma de datos se llevó a cabo durante tres meses y dos semanas.

\section{Análisis y discusión}

Para cumplir con el objetivo de caracterizar los procesos metacognitivos que ocurrieron en el desarrollo de la secuencia didáctica, se construyeron tres categorías que corresponderán a los distintos apartados que siguen a continuación. Se definirá cada una de ellas y se las ilustrará con algún ejemplo asociado a los dos modos de razonar que explicitamos antes: el esencialismo y el determinismo.

\section{Señalamiento del obstáculo epistemológico}

En esta categoría se incluyen aquellas expresiones en las que los estudiantes señalan que bajo alguna explicación subyace el obstáculo epistemológico. Es un tipo de regulación simple, en contrapartida con una regulación compleja como la que se mostrará en los siguientes apartados. 
A continuación proponemos dos ejemplos. El primero de ellos corresponde a la actividad 8, donde los estudiantes debían identificar los modos de pensar subyacentes en algunas explicaciones iniciales de estudiantes del mismo grupo-clase. En el segundo se les proponía revisar una explicación de un alumno ficticio, marcando en ella las frases que decidieran corregir.

[Mis compañeros estaban pensando que] Todos son iguales, mutan para sobrevivir, van a mutar todos, mutan para sobrevivir. (Olivia, $2^{\circ} 2^{\circ}$, Actividad 8.1)

Frase del alumno ficticio corregida "y los lobos con poco pelo no sobrevivieron y se murieron". No se murieron todos (pensamiento extremitista) (Juliana, $2^{\circ} 2^{\circ}$, Actividad 9.1)

Para el caso de Olivia, propone que las explicaciones iniciales de algunos estudiantes de su grupo-clase asumían que “Todos son iguales”. Inferimos que aquí se está señalando el esencialismo dado que despreciar la variabilidad entre los organismos es uno de los supuestos de este modo de razonar. Si bien la estudiante no menciona explícitamente el obstáculo epistemológico, consideramos que la aclaración que realiza corresponde a uno de los obstáculos epistemológicos que se han trabajado en la secuencia didáctica. En este sentido no sería una expresión azarosa, sino que más bien daría cuenta de un señalamiento del obstáculo.

Por otro lado, en el caso de Juliana sí se hace explícito el señalamiento del determinismo. La alumna identifica al "pensamiento extremitista", que fue la etiqueta lingüística consensuada por el grupo clase para llamar al determinismo. En el grupo-clase de esta alumna se discutió oralmente la funcionalidad del modo de razonar determinista, la transversalidad del mismo y su conflictividad con los modelos que se estaban construyendo. Posteriormente, se consensuó con todo el grupo-clase dicha etiqueta, para llamar a este modo de razonar. El alumno que propuso dicho nombre indicó que era "Porque estás en un extremo o en el otro" ( $2^{\circ} 2^{\circ}$, Actividad 6, Puesta en Común 07:00). Esto surgió de la discusión de un caso de evolución de los lobos a través del tiempo. En dicho caso se ponía en discusión si cabían otras posibilidades entre los extremos de morir o sobrevivir, para un lobo de pelaje específico.

Sintetizando, podemos mencionar dos tipos de señalamiento de los obstáculos epistemológicos. Uno de tipo implícito, en el que los estudiantes realizan aclaraciones que aluden al obstáculo epistemológico pero sin nombrarlo directamente. Otro de tipo explícito, donde los estudiantes mencionan que a la explicación subyace algún modo de pensar, el cual puede haber sido nombrado de manera explícita durante las clases.

\section{Regulación individual compleja}

La regulación individual de los obstáculos epistemológicos implica un señalamiento del obstáculo (de manera implícita o explícita) así como un ensayo de explicación alternativa con elementos construidos durante el proceso de modelización.

Llamamos complejas a estas regulaciones en el sentido de que, además de señalar el obstáculo, se incluye una explicación alternativa con elementos que se construyeron durante la secuencia didáctica. Este tipo de regulaciones es individual en tanto que los estudiantes someten la regulación a sus propias explicaciones.

A continuación, mostramos dos ejemplos de esta categoría. ${ }^{2}$ Los mismos corresponden a una actividad hacia la mitad de la secuencia didáctica. En la primera parte de ella, se

2 Los ejemplos muestran la transcripción fiel de los textos de los estudiantes, incluyendo algunas frases que fueron tachadas por ellos. 
pedía a los estudiantes construir una primera aproximación a una base de orientación para la resolución de problemas en los que hubiese que aplicar el modelo de selección natural (ejemplo 1). En la segunda parte de esta actividad, se les solicitaba a los estudiantes explicar el cambio de los lobos a través del tiempo (ejemplo 2).

En la SN: Los antepasados de una especie sobreviven porque nacieron con cualidades favorables (todos los individuos de una especie son diferentes); Los cambios genéticos son al azar, no cambian para algo. Las variaciones no aparecen según las necesidades de los individuos ("Pensamiento con motivo"); Los individuos con cualidades no favorables tienen menos probabilidad de sobrevivir ("Pensamiento extremitista"); Recordar que nadie selecciona los individuos; Objetivo: dejar descendencia; No olvidar que el ambiente modifica a la población. (Lucio, $2^{\circ} 2^{\circ}$, Actividad 9.2)

Los individuos de la población de sus antecesores son diferentes, algunos tienen mucho pelo y otros menos. El gran pelaje les permite sobrevivir en zonas árticas por lo cual es una característica favorable respecto al ambiente, lo que les permite sobrevivir y reproducirse más que los otros que no poseen esa característica. A través del tiempo, luego de varias generaciones hubo más lobos con un gran pelaje, ya que no todos los individuos se adaptan. Es por esta razón que actualmente los lobos árticos tienen un gran pelaje. (Eliana, $2^{\circ} 1^{\circ}$, Actividad 9.3)

Es importante recordar que las bases de orientación son instrumentos que sirven como modo de planificar (García Rovira y Sanmartí, 1998), en este caso la construcción de explicaciones a partir del modelo científico. Lucio planifica de manera explícita el encuentro con el obstáculo determinista como modo de razonar que está presente en la construcción de explicaciones evolutivas. Dicha planificación la realiza a partir de mencionar la etiqueta consensuada por el grupo-clase para llamar al determinismo "Pensamiento extremitista". Además incluye una explicación alternativa al determinismo, aludiendo a la probabilidad, cuando indica: "Los individuos con cualidades no favorables tienen menos probabilidad de sobrevivir".

En el segundo ejemplo presentado, se transcribe la explicación de Eliana. En ella se encuentra un señalamiento implícito del esencialismo al indicar que " no todos los individuos se adaptan", esto habilita pensar que existen diferentes individuos y que además pueden tener distintos destinos. Así la explicación alternativa que construye la alumna puede caracterizarse por: Pensar en términos poblacionales ("Los individuos de la población de sus antecesores son diferentes, algunos tienen mucho pelo y otros menos") y en una reproducción diferencial ("les permite sobrevivir y reproducirse más que los otros que no poseen esa característica"). Dos de los conceptos claves del modelo de evolución por selección natural, construidos durante las actividades de la secuencia didáctica.

En estos casos quien regula es el propio sujeto, y lo hace a sí mismo, a sus propias construcciones/explicaciones. Esta regulación, como ya hemos indicado, implica considerar un señalamiento del obstáculo (de manera implícita o explícita) y un ensayo de explicación alternativa.

\section{Regulación social compleja}

Definimos la regulación social de los obstáculos epistemológicos como aquella regulación que se realiza sobre producciones ajenas, de individuos reales (durante la construcción de una explicación colaborativa) o ficticios (presentados por el docente). $\mathrm{Al}$ igual que en el caso anterior, esta regulación incluye un señalamiento (explícito o implícito) de una producción ajena, bajo la que subyace algún modo de razonamiento y una explicación alternativa utilizando diversos elementos construidos durante las actividades de la secuencia didáctica. 
El ejemplo que presentamos a continuación corresponde a una discusión grupal de los estudiantes durante una actividad en la que debían reformular un esquema conceptual que habían realizado al inicio de la secuencia didáctica. Dicho esquema conceptual representaba una explicación inicial sobre cómo creían los estudiantes que habían evolucionado un grupo de lobos a través del tiempo.

\begin{tabular}{ll}
\hline Olivia & $\begin{array}{l}\text { Yo tacharía “Todos los lobos cambian el pelaje”y pondría un individuo es } \\
\text { diferente, digamos, un individuo es diferente a los demás... }\end{array}$ \\
\hline Francesca & No entiendo lo que querés decir. \\
\hline Olivia & Que un individuo es diferente, con otro pelaje... \\
\hline Lucas & $\begin{array}{l}\text { Tiene características diferentes [...] Hay que pensar siempre que son todos } \\
\text { diferentes. }\end{array}$ \\
\hline Olivia & Claro. \\
\hline Lucas & Nadie es igual. \\
\hline Olivia & Entonces “Todos” no sería. Lo tacho. \\
\hline Lucas & Si. Sería... algunos. Algunos sería acá. Algunos lobos... \\
\hline Olivia & Algunos lobos tienen cualidades diferentes. \\
\hline Lucas & Si, es lo mismo cualidad y característica. \\
\hline Ignacio & No, todos tienen características... \\
\hline Olivia & Algunos lobos son más aptos żqué pongo? ¿Tienen características ventajosas? \\
\hline Francesca & Eh... \\
\hline Lucas & Alobos tienen sus propias características. Cada uno tiene sus formas de pelaje... \\
\hline
\end{tabular}

$\left(2^{\circ} 2^{\circ}\right.$, Actividad Reguladora 2, Grupo 3, 07:38)

En la discusión Olivia propone tachar del esquema conceptual inicial la frase "Todos los lobos cambian el pelaje". Frente al no entendimiento de Francesca, Lucas propone que "Hay que pensar siempre que son todos diferentes". En este momento está haciendo explícita la necesidad de pensar en la variabilidad poblacional, lo que inferimos se trata de una regulación del esencialismo. A partir de allí la construcción de estos tres estudiantes versa sobre las diferentes características de los organismos que responderían a su comprensión sobre la variabilidad poblacional.

En este ejemplo que presentamos, un individuo regula una explicación que se está construyendo de manera colectiva, anticipando la necesidad de pensar en términos distintos al obstáculo epistemológico. Como hemos indicado, esta regulación implica un señalamiento del obstáculo y una explicación alternativa que se construye a partir de elementos trabajados durante la secuencia.

Si bien estas actividades, donde aparece la regulación social, se encuentran enmarcadas en una secuencia basada en la regulación de los obstáculos epistemológicos, las mismas no tenían la finalidad de que los estudiantes regularan los obstáculos. Esto sucedió espontáneamente anclado a la construcción de una explicación en el marco de dichas actividades. 


\section{Conclusiones}

La enseñanza y el aprendizaje de los modelos de las ciencias puede ser una tarea dificultosa. El eje de este trabajo versó sobre ciertos modos de razonar (como el esencialismo o el determinismo) que pueden pensarse como obstáculos a sortear durante los procesos de construcción de explicaciones en el aula. Dado que no es posible eliminar estos modos de razonar -recordemos que son funcionales para los sujetos en ciertos momentos de su cotidianeidad- lo que se propone es el desarrollo de una conciencia y una regulación (metacognición) sobre los mismos.

En este trabajo nos propusimos caracterizar los procesos de regulación metacognitiva que emergieron durante una propuesta didáctica basada en la modelización y la metacognición sobre los obstáculos epistemológicos. En el marco de la enseñanza de dos modelos de la biología evolutiva, caracterizamos tres modos de regular:

(1) Señalamiento del obstáculo epistemológico. En estas expresiones los estudiantes señalan que bajo alguna explicación subyace un obstáculo epistemológico. Esto puede ocurrir de manera implícita (sin aludir a este modo de pensar pero refiriéndose a algunas de sus consecuencias) o explícita (refiriéndose de manera manifiesta al modo de razonar).

(2) Regulación individual compleja. Los estudiantes señalan el obstáculo sobre sus propias explicaciones (muchas veces como una aclaración) y a su vez ensayan una explicación alternativa con elementos construidos durante el proceso de modelización.

(3) Regulación social compleja. En ella los estudiantes regulan producciones ajenas a partir de un señalamiento del obstáculo (explícito o implícito) y una explicación alternativa utilizando diversos elementos construidos durante las actividades de la secuencia didáctica.

Consideramos que esta caracterización de las regulaciones puede ayudar a pensar en actividades útiles para mejorar el desarrollo de las habilidades metacognitivas. Recordemos que estas habilidades son un elemento necesario, aunque no el único, para que los estudiantes desarrollen la capacidad de pensamiento crítico. 


\section{Bibliografía}

"Astolfi, J.y Peterfalvi, B. (2001). “Estrategias para trabajar los obstáculos: dispositivos y resortes”. En Camilloni, A. (Comp.), Los obstáculos epistemológicos en la enseñanza (pp. 191-223). Barcelona: Gedisa.

»Astolfi, J. P. (2003). Aprender en la escuela. Chile: Comunicaciones Noreste Ltds.

"Atran, S.; Medin, D.; Lynch, E.; Vapnarsky, V.; Ucan Ek, E. y Sousa, P. (2001). Folkbiology doesn't come from folkpsychology: Evidence from Yukatek Maya in cross-cultural perspective. Journal of Cognition and Culture, 1(1), pp. 3-42.

» Carretero, M. (2009). Constructivismo y educación. Buenos Aires: Paidós.

"Coley, J. y Muratore, T. (2012). "Trees, Fish, and Other Fictions. Folk biological thought and its implicationes for understanding evolutionary biology". En Rosengren, K.; Brem, S.; Evans, E. y Sinatra, G. (Eds.), Evolution Challenges. Integrating research and practice in teaching and learning about evolution (pp. 22-46). Nueva York: Oxford University Press.

"Creswell, J. (2012). Educational research. Planning, conducting, and evaluating quantitative and qualitative research. Boston: Pearson.

»Del Río, M. F. y Strasser, K. (2007). ¿Tienen los niños una teoría esencialista acerca de la pobreza? Psykhe, 16 (2), pp. 139-149.

»Dennett, D. (1999). La peligrosa idea de Darwin. Evolución y significados de la vida. Barcelona: Galaxia Gutenberg.

»Dupré, J. (2006). El legado de Darwin. Qué significa hoy la evolución. Buenos Aires: Katz.

"Evans, E. (2008). "Conceptual change and evolutionary biology: A developmental analysis". En Vosniadou, S. (Ed.), International handbook of research on conceptual change (pp. 263-294). Nueva York: Routledge.

"Evans, E. y Rosengren, K. (2018). “Cognitive biases or cognitive bridges?”. En Kampourakis, K. (Ed.), Teaching Biology in Schools (pp. 9-21). Nueva York: Routledge.

"Fletcher, L. y Carruthers, P. (2012). Metacognition and reasoning. Philosophical Transactions of the Royal Society B: Biological Sciences, 367 (1594), pp. 1366-1378.

» Flick, U. (2007). Introducción a la investigación cualitativa. Madrid: Morata.

"Ford, C. y Yore, L. (2012). “Toward convergence of critical thinking, metacognition, and reflection: illustrations from natural and social science, teacher education and classroom practice". En Zohar, A. y Dori, Y. (Eds.), Metacognition in Science Education. Trends in Current Research (pp. 251-271). Nueva York: Springer.

» Futuyma, D. (2009). Evolution. Oxford: Sinauer.

» García, J. E. (1998). Hacia una teoría alternativa sobre los contenidos escolares. Sevilla: Díada.

》 García Rovira, M. P. y Sanmartí, N. (1998). Las bases de orientación: un instrumento para enseñar a pensar teóricamente en biología. Alambique: Didáctica de las Ciencias Experimentales, 16, pp. 8-20.

» Gelman, S. y Rhodes, M. (2012). “Two-Thousand Years of Stasis. How psychological essentialism impedes evolutionary understanding”. En Rosengren, K.; Brem, S.; 
Evans, E. y Sinatra, G. (Eds.), Evolution Challenges. Integrating research and practice in teaching and learning about evolution (pp. 3-21). Nueva York: Oxford University Press.

»Glaser, B. y Strauss, A. 1967. The discovery of Grounded Theory strategic for qualitative research. Nueva York: Aldine Publishing Company.

» González Galli, L. (2011). Obstáculos para el aprendizaje del modelo de evolución por selección natural (Tesis doctoral). Universidad de Buenos Aires, Facultad de Ciencias Exactas y Naturales, Buenos Aires.

» González Galli, L. y Meinardi, E. (2015). Obstáculos para el aprendizaje del modelo de evolución por selección natural en estudiantes de escuela secundaria de Argentina. Ciencia y Educação, 21 (1), pp. 101-122.

» González Galli, L. y Meinardi, E. (2016). “Obstáculos para el aprendizaje del modelo de evolución por selección natural”. En Cuvi, N.; Sevilla, E.; Ruíz, R. y PuigSamper, M. (Eds.), Evolucionismo en América y Europa (pp. 463-476). Ecuador: Centro Publicaciones PUCE.

» González Galli, L. y Meinardi, E. (2017). Obstáculos para el aprendizaje del modelo de evolución por selección natural en estudiantes universitarios de biología. Revista Eureka sobre Enseñanza y Divulgación de las Ciencias, 14 (3), pp. 435-449.

"González Galli, L.; Pérez, G.; Alegre, C. y Joelson, S. (2018). Explicaciones, concepciones y obstáculos sobre el origen de las especies en estudiantes de Escuela Secundaria de Argentina. Ciência \& Educação, 24 (3), pp. 741-758.

"Gregory, T. (2009). Understanding natural selection: essential concepts and common misconceptions. Evolution: Education and Outreach, 2 (2), pp. 156-175.

» Halpern, D. (2013). Thought and knowledge: An introduction to critical thinking. Nueva York: Psychology Press.

" Hirschfeld, L. (2002). “¿La adquisición de categorías sociales se basa en una competencia dominio-específica o en la transferencia de conocimientos?". En Hirschfeld, L. y Gelman, S. (Comps.), Cartografía de la mente: la especificidad de dominio en la cognición y en la cultura (pp. 285-328). Barcelona: Gedisa.

» Hogan, M.; Dwyer, C.; Harney, O.; Noone, C. y Conway, R. (2015). “Metacognitive Skill Development and Applied Systems Science: A Framework of Metacognitive Skills, Self-regulatory Functions and Real-World Applications". En Peña-Ayala, A. (Ed.), Metacognition: Fundaments, Applications, and Trends. A Profile of the Current State-OfThe-Art (pp. 75-106). Suiza: Springer.

» Johsua, S. y Dupin, J. (2005). Introducción a la didáctica de las ciencias y la matemática. Buenos Aires: Colihue.

》 López Aymes, G. (2012). Pensamiento crítico en el aula. Docencia e Investigación, 37 (22), pp. 41-6o.

» Martinand, J. L. (1986). Enseñanza y aprendizaje de la modelización. Enseñanza de las Ciencias, 4, pp. 45-50.

" Mayr, E. (2006). “Typological versus Population Thinking”. En Sober, E. (Ed.), Conceptual Issues in Evolutionary Biology (pp. 326-328). Massachusetts: The MIT Press.

»Mead, L. y Scott, E. (2010). Problem Concepts in Evolution Part II: Cause and Chance. Evolution: Education and Outreach, 3, pp. 261-264.

» Norabuena, G.; Velazquez-Tejeda, M. y Hernández, R. (2017). Estrategias innovadoras para contribuir al desarrollo del pensamiento crítico. Revista de Educación, o (10), pp. 31-6o. 
»Pérez G., Gómez Galindo, A. y González Galli, L. (2018). Enseñanza de la evolución: fundamentos para el diseño de una propuesta didáctica basada en la modelización y la metacognición sobre los obstáculos epistemológicos. Revista Eureka sobre Enseñanza y Divulgación de las Ciencias, 15 (2), 2102.

» Peterfalvi, B. (2001). “Identificación de los obstáculos por parte de los alumnos”. En Camilloni, A. (Comp.), Los obstáculos epistemológicos en la enseñanza (pp. 127-168). Barcelona: Gedisa.

» Pozo, J. (2014). Psicología del Aprendizaje Humano: Adquisición de conocimiento y cambio personal. Madrid: Morata.

» Pozo, J. (2016) Aprender en tiempos revueltos. La nueva ciencia del aprendizaje. Madrid: Alianza.

»Puig, B. y Jiménez Aleixandre, M. P. (2015). El modelo de expresión de los genes y el determinismo en los libros de texto de ciencias. Revista Eureka sobre Enseñanza y Divulgación de las Ciencias, 12 (1), pp. 55-65.

»Solbes, J. (2013). Contribución de las cuestiones sociocientíficas al desarrollo del pensamiento crítico (I): Introducción. Revista Eureka sobre Enseñanza y Divulgación de las Ciencias, 10 (1), pp. 1-10.

» Sperber, D. (2002). “La modularidad del pensamiento y la epidemiología de las representaciones”. En Hirschfeld, L. y Gelman, S. (Comps.), Cartografía de la mente: la especificidad de dominio en la cognición y en la cultura (pp. 71-107). Barcelona: Gedisa.

»Stamos, D. (2008). Evolución. Los grandes temas: sexo, raza, feminismo, religión y otras cuestiones. Barcelona: Biblioteca Buridán.

» Strauss, A. y Corbin, J. (1990). Basics of Qualitative Research: Grounded Theory Procedures and Techniques. Newbury Park: Sage.

" Tamayo Alzate, O. (2014). “Didácticas dominio-específicas y modularidad de la mente”. En Mosquera Suárez, C. (Comp.), Miradas Contemporáneas en Educación № 2: Algunos puntos clave para el debate (pp. 83-108). Colombia: Universidad Distrital Francisco José de Caldas.

» Tamayo Alzate, O.; Zona López, J. y Loaiza Zuluaga, Y. (2017). La metacognición como constituyente del pensamiento crítico en el aula de ciencias. Tecné, Episteme y Didaxis, Número extraordinario, pp. 1031-1036.

" Torres, N. ySolbes, J. (2018). “Pensamiento crítico desde cuestiones socio-científicas”. En Conrado, D. y Nunes-Neto, N. (Eds.), Questoes sociocientíficas - Fundamentos, propostas de ensino e perspectivas para acoes sociopolíticas. Salvador: Edufba.

» Tversky, A. y Kahneman, D. (1974). Judgment under uncertainty: Heuristics and biases. Science, 185 (4157), pp. 1124-1131.

"Waxman, S.; Medin, D. y Ross, N. (2007). Folkbiological reasoning from a crosscultural developmental perspective: early essentialist notions are shaped by cultural beliefs. Developmental Psychology, 43 (2), pp. 294-308.

\section{Gastón Mariano Pérez}

Doctorando en Biología, Universidad de Buenos Aires. Profesor de enseñanza media y superior en Biología, UBA. Becario doctoral, Consejo Nacional de Investigaciones Científicas y Técnicas, UBA. gastonperezbio@gmail.com 


\section{Leonardo Martín González Galli}

Doctor en Biología, Universidad de Buenos Aires. Profesor de enseñanza media y superior en Biología, UBA. Investigador Adjunto del Consejo Nacional de Investigaciones Científicas y Técnicas, UBA. leomgalli@gmail.com 